\title{
Effects of Acute and Chronic Alprazolam Treatment on Cerebral Blood Flow, Memory, Sedation, and Plasma Catecholamines
}

\author{
Peter Roy-Byrne, M.D., Joseph Fleishaker, Ph.D., Carroll Arnett, Ph.D., \\ Mark Dubach, Ph.D., Jodi Stewart, B.S., Allen Radant, M.D., Richard Veith, M.D., \\ and Michael Graham, M.D., Ph.D.
}

The effects of $0.014 \mathrm{mg} / \mathrm{kg}$ intravenous alprazolam dministration on cerebral blood flow (CBF), memory, sdation, and plasma norepinephrine and epinephrine are determined in eight healthy males at baseline levels and following 1 week of daily oral alprazolam treatment. As baseline, intravenous alprazolam administration oused acute reductions in whole-brain CBF (25\% to $30 \%$ decrease), memory, plasma epinephrine, and selfmed alertness. Following 1 week of alprazolam treatment, tolerance developed to the acute effects of intravenous alprazolam on CBF, memory, and plasma epinephrine. There were no consistent regional neuroanatomic differences in the CBF effects of acute alprazolam, or in the development of tolerance to these effects, and no correlations between the various measures of acute alprazolam effects on either test day.

[Neuropsychopharmacology 8:161-169, 1993]
Ir wORDS: Cerebral blood flow; Alprazolam;

Rrzodiazepines; Tolerance; Catecholamines

lenzodiazepines are widely prescribed for the treatment of anxiety, panic attacks, and insomnia. Their multiple anxiolytic, sedative, myorelaxant, ataxic, and mnestic effects are mediated by specific receptors that ue allosterically linked to gamma aminobutyric acid (GABA)-A receptors and potentiate neuronal inhibition indiverse brain regions (Roy-Byrne and Nutt 1991). Although their wider range of safety has made benzodiaxpines the preferred class of hypnotic/anxiolytic

From the Departments of Psychiatry and Behavioral Sciences (PRB, C. MD, AR, RV) and Radiology (JS, MG), University of Washington, Seatle; the Veterans Affairs Medical Center (AR, RV), Seattle, Westington; and the Clinical Pharmacokinetics Unit (JF), The UpJohn Company, Kalamazoo, Michigan.

Address reprint requests to: Peter Roy-Byrne, M.D., Chief of Aychiatry, Harborview Medical Center ZA-15 325 9th Ave., Seattle, Wrshington 98104.

Received February 3, 1992; revised April 20, 1992; accepted May 9.1992. drugs, renewed concernsabout the occurrence of abuse, tolerance, and dependence with long-term dosing has resulted in recent increased regulation of benzodiazepine (Brahams 1990).

Although numerous studies have examined the acute pharmacodynamic and pharmacokinetic effects of benzodiazepines in normal and anxious human subjects, fewer studies have examined the effects of chronic administration of benzodiazepines in human subjects, beyond their therapeutic efficacy in anxious patients (reviewed in McLeod et al. 1988). Relatively little work has been done directly measuring the effects of benzodiazepines in neuroanatomically discrete brain regions. Three studies have examined the acute effects of benzodiazepines on cerebral blood flow (CBF) (Mathew et al. 1985, 1991) and glucose metabolism (de Witt et al. 1991) in normal human subjects, and one study has examined the effect of 3 weeks of chronically administered benzodiazepine on cerebral glucose metabolism in anxious human subjects (Buchsbaum et al. 1987).

This study investigated the acute intravenous and 
chronic (oral) effects of currently the most widely prescribed benzodiazepine, alprazolam, on CBF measured by positron-emission tomography (PET), memory, sedation, and plasma catecholamines (norepinephrine and epinephrine). By repeating the acute alprazolam challenge after 1 week of continuous treatment with oral alprazolam, we examined the development of tolerance using multiple pharmacodynamic measures and investigated whether there were regional neuroanatomic differences in the development of tolerance to the CBF effects of acute alprazolam administration.

\section{METHODS}

\section{Subjects}

Eight right-handed males ranging in age from 18 to 25 years were recruited by advertisement in the University of Washington Health Sciences Center. Volunteers were employees or students in the health sciences. They were in good mental and physical health currently and by history. Psychiatric history was obtained by Structured Clinical Interview for DSM-III-R (SCID), volunteer version. Patients had not taken medication for the past month and had no history of exposure to benzodiazepines or other psychotropic medications. Alcohol use histories indicated three subjects never drank, two drank rarely (once per month), and three drank on a more regular "social" basis (two to three times weekly) but did not meet SCID criteria for alcohol abuse or dependence.

\section{Experimental Design}

The design of the study is presented in Figure 1. Subjects ingested nothing by mouth after midnight on the night before testing and came to the PET suite at $8 \mathrm{~A} . \mathrm{M}$. for insertion of arterial lines, PET slice alignment with magnetic resonance imaging (MRI) and transmission scan. Baseline measures of CBF, sedation, memory, and plasma norepinephrine were obtained, and 30 to 60 minutes later subjects were given $0.014 \mathrm{mg} / \mathrm{kg}(0.045$ mmole $/ \mathrm{kg}$ ) alprazolam intravenously over 60 seconds. All measures were repeated 15,45 , and 75 minutes after injection. Subjects then began taking alprazolam 0.5 $\mathrm{mg}(1.6 \mathrm{~mol})$ orally 4 times a day $(0.25 \mathrm{mg} 4$ times daily for the 1st day and one-half) for 7 days. On the 8th day, they took their $7 \mathrm{~A} . \mathrm{M}$. alprazolam dose $(0.5 \mathrm{mg})$, came to the PET suite at $8 \mathrm{~A} . \mathrm{M}$. , and the entire procedure, including acute intravenous alprazolam administration, was repeated.

\section{Positron-Emission Tomography Conditions}

All the PET studies were done with a Scanditronix SP. 3000 , which had been considerably modified. This tomograph is a whole-body time-of-flight machine with $\mathrm{BaF}$ crystals that is particularly well suited for the high count rate studies done with bolus injection of $\mathrm{O}^{15} \mathrm{wa}$ ter. The images were reconstructed with a resolution of $10 \mathrm{~mm}$.

The subjects were positioned carefully, using a lateral skull $x$-ray, and the head was constrained with a customized polyurethane foam head holder and thermoplastic face mask. Prior to injection of any tracer, an attenuation data set was acquired with a rotating $\mathrm{Ge}-68$ source for 40 minutes. This was used later during image reconstruction to correct the emission data appropriately. Before the attenuation scan, an intravenous catheter was placed in the antecubital fossa on the right and a radial arterial catheter was placed on the left. A calibration vial containing $\mathrm{Ge}^{68}$ was in the field of view and an aliquot from the vial was counted in the gamma counter for cross-calibration between the counter and
Figure 1. The overall experimental design is schematically depicted.

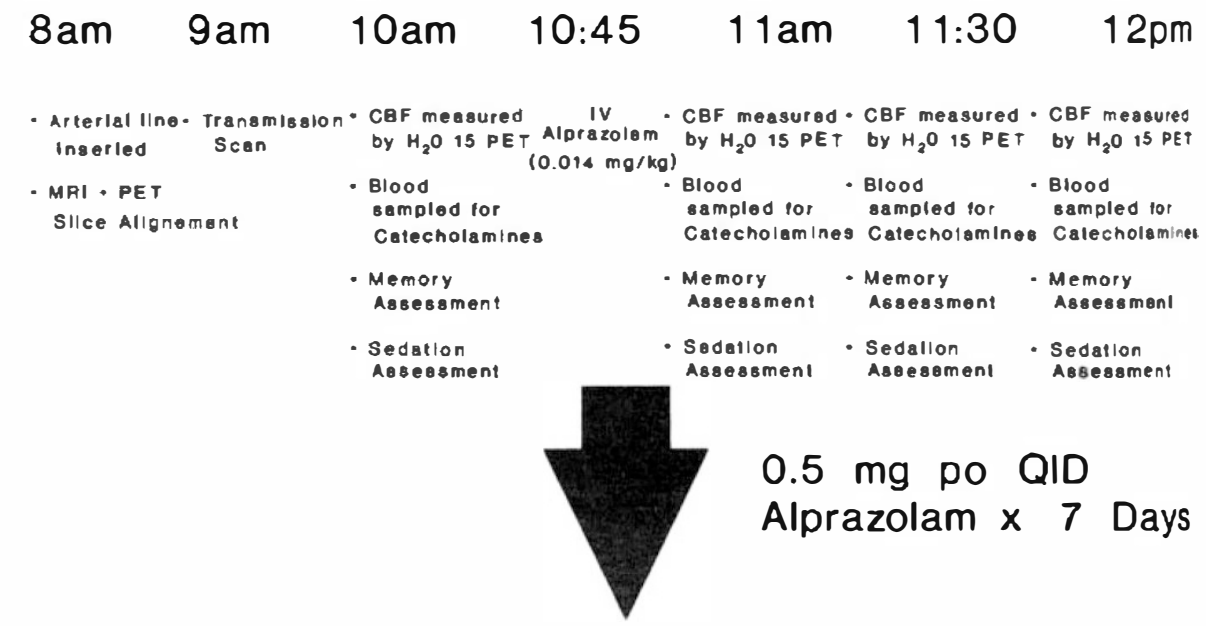

REPEAT ABOVE PROCEDURE 
the tomograph. Scans were performed in dim light with the subject's eyes open and low-level background noise. The first PET scan followed at about 10 A.M. Beginning 30 seconds prior to intravenous injection of 40 to $50 \mu \mathrm{Ci}$ (1480 to $1850 \mathrm{MBq}$ ) $\mathrm{H}_{2}{ }^{15} \mathrm{O}$, and continuing for the 2 minutes that data was acquired during each PET scan, subjects performed a simple visual continuousperformance task by pressing a button with their right hand whenever the letter " $B$ " appeared on a computer screen overhead (A, B, C, and D were shown in random order, one letter every 3 seconds). This task assured that subjects were awake during the scan and subjects were also kept awake between scans by being engaged in emotionally neutral conversation. Arterial blood samples were obtained every 2 seconds via an automated blood sampling machine (Graham and lewellen 1988) and a final sample for plasma catecholamines and alprazolam level was obtained at the end of the2-minute scan. Cerebral blood flow values were calwlated using the Kety method modified by Herscovitch with a permeability surface area product for water of $1.04 \mathrm{~m} / \mathrm{gm} / \mathrm{min}$ (Herscovitch et al. 1984).

\section{Positron-Emission Tomography-Magnetic Resonance Imaging}

On the day prior to a study, the subject came to the PET suite and had the head holder and thermoplastic mask molded to fit his face. He was taken to the MRI suite where a sagittal MRI was obtained, and from it the oblique transverse plane containing the intercommissural (AC-PC) line was determined. A plastic card, aped to the forehead of the mask, bearing three evenly spaced oil-filled tubes, provided landmark points on the sagittal MRI. Three parallel MRI slices were obtained, $7.2 \mathrm{~mm}$ below and $7.2 \mathrm{~mm}$ and $21.6 \mathrm{~mm}$ above the AC-PC plane. These slices were selected to include neuroanatomic areas rich in benzodiazepine and/or implicated by preclinical autoradiographic studies of benzodiazepine effects (Ableitner et al. 1985; Nehlig et al. 1987), including neocortex, striatum, thalamus, midtrain, and cerebellum. On the next day, in the PET suite, the PET gantry was rotated to bring PET slices parallel to the oblique transverse AC-PC plane, and the able was moved axially to bring the three PET planes (on $14.4 \mathrm{~mm}$ centers) into register with the MRI planes. Lateral skull $x$-rays were used to confirm the position, using as landmarks the sella turcica and a metal-rod replica of the MRI landmarks taped to the mask. In this manner, coregistration error was reduced to slippage of the rest position of the skull in the tightly fitted mask. figure 2 depicts the three MRI slices of one subject, showing all regions of interest obtained.

\section{Other Measures}

Memory effects were measured using a simple wordlist method developed by Weingartner and validated as being benzodiazepine sensitive in prior studies (RoyByrne et al. 1987). Four tasks measured immediate recognition memory, free recall, and delayed recognition of twelve categorically similar words, six of which were repeated twice (perfect score $=36$ ). Self-rated sedation was measured by asking patients to respond orally to five separate 0 to 100 analogue-type scales using different synonyms for alert (0) and drowsy (100). This oral modification of a well-validated visual analogue scale method (Bond and Lader 1974) has not been validated and may have less sensitivity to benzodiazepine effects than the visual scale, but was necessary since subjects had to remain in the scanner and were unable to manipulate pen and paper. The mean score for the five scales was used as the measure. Plasma norepinephrine and epinephrine were measured in duplicate using a single-isotope enzymatic assay (Evans et al. 1987), and plasma alprazolam levels were measured by highperformance liquid chromatography (Smith and Kroboth 1987).

\section{Statistical Analysis}

Statistical analysis was done to determine separate within-session drug effects for each test day and then to compare within-session effects across days. One-way repeated-measures analysis of variance (ANOVA) was performed for each measure on the data from Day 1 , comparing four sets of values grouped by time interval $(0,15,45$, and 75 minutes after injection); a significant effect of time interval was interpreted as indicating an alprazolam effect. The same analysis was performed on the data from Day 8.

Two-way repeated-measures ANOVA was also performed for the same data from both days, comparing eight sets of values grouped by time interval and by day; a significant interaction between time interval and day indicated that the observed drug effect (changeover time compared to baseline) was significantly different on the two days, suggesting tolerance to alprazolam's effect.

In addition, the two sets of baseline (time interval 0) values for each measure were compared by ANOVA; a significant decrease on Day 8 as compared to Day 1 was interpreted as a possible indication of a "floor" effect that could prevent a further acute drop in the value, contributing to the appearance of alprazolam tolerance on Day 8.

Analyses were repeated for $\mathrm{CBF}$ for individual neuroanatomic regions, with raw $\mathrm{CBF}$ values "normalized" to the whole-slice $\mathrm{CBF}$ (i.e., region $\mathrm{CBF} /$ slice $\mathrm{CBF}$ ), to factor out contributions of global $\mathrm{CBF}$ changes to individual regional changes.

For Day 1, for each measure, the maximum change from baseline observed at the three subsequent time intervals was calculated; correlation coefficients comparing these maximal effects for every pairing of measures were calculated to determine whether the mea- 
Figure 2. The three horizontal slice levels on MRI are depicted with the individual neuroanatomic regions of interest. Slices are parallel to the AC-PC line, $7 \mathrm{~mm}$ below and $7 \mathrm{~mm}$ and $21 \mathrm{~mm}$ above it. Large print indicates lateral regions and small print more centrally located regions.

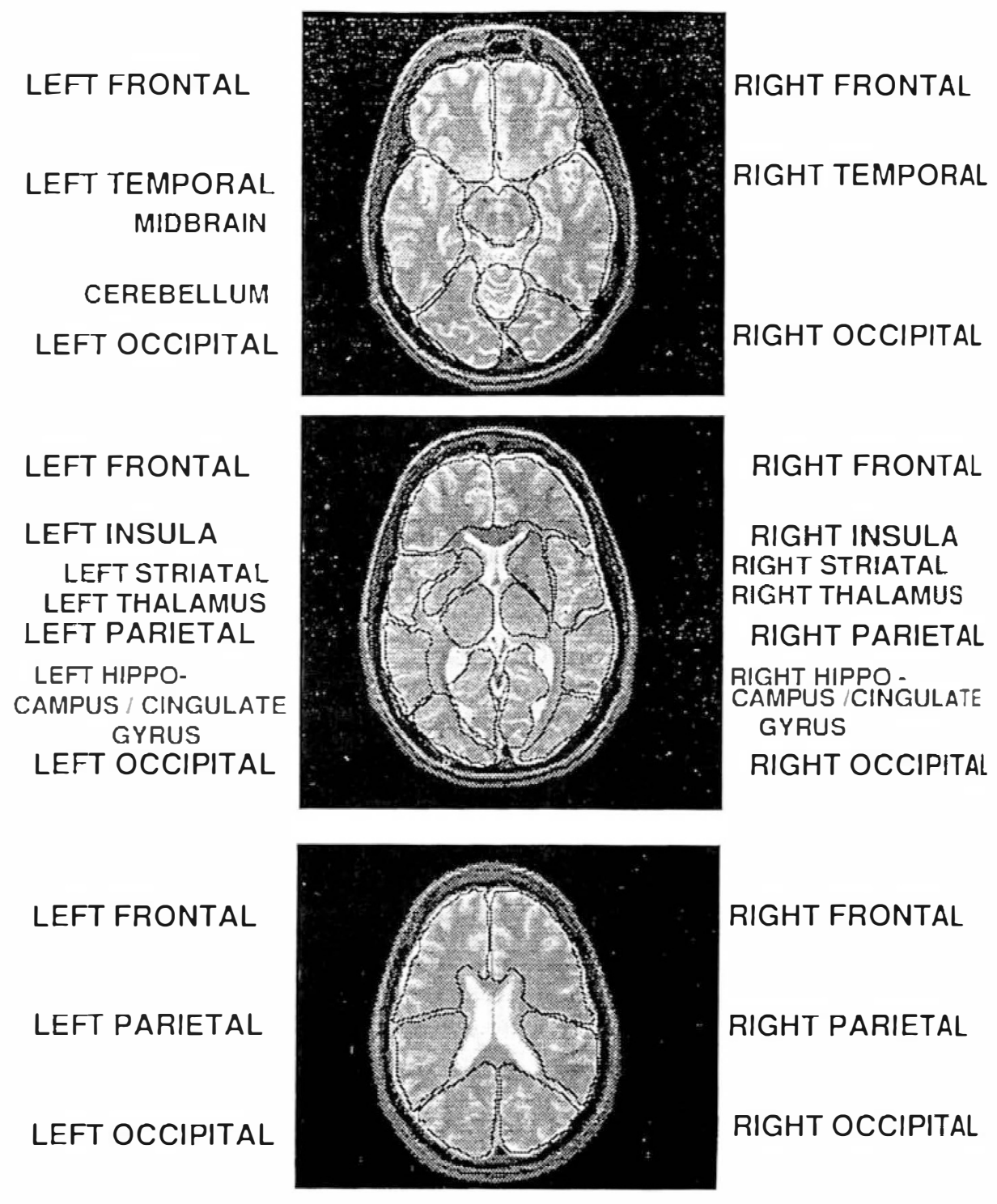

\section{LEFT FRONTAL \\ LEFT TEMPORAL MIDBRAIN \\ CEREBELLUM \\ LEFT OCCIPITAL \\ LEFT FRONTAL \\ LEFT INSULA \\ LEFT STRIATAL LEFT THALAMUS LEFT PARIETAL \\ LEFT HIPPO- \\ / CINGULATE GYRUS \\ LEFT OCCIPITAL}

\section{LEFT FRONTAL \\ LEFT PARIETAL \\ LEFT OCCIPITAL}

\author{
RIGHT FRONTAL \\ RIGHT INSULA \\ RIGHT STRIATAL \\ GHT THALAMUS \\ CAGT HIPPO \\ CAMPUS /CINGULATE \\ GYRUS \\ RIGHT OCCIPITAL
}

RIGHT FRONTAL

RIGHT PARIETAL

RIGHT OCCIPITAL sures were independent. The same procedure was followed for Day 8 and for the difference between Day 1 and Day 8.

Complete data on CBF was available for only six of eight subjects. Data for one subject on Day 1 was lost during computer transformation of raw activity counts so that Day 8 data was of little use. For another subject, the CBF value on Day 1 baseline was judged to be invalid, being only $60 \%$ of the value of all other subjects' Day 1 baselines. This resulted in this subject's subsequent $C B F$ values obtained after alprazolam being substantially higher than this baseline (in contrast to all other subjects). Conversely, behavioral, cognitive, and catecholamine effects for this subject were similar to those of all other subjects (i.e., in the opposite direction from CBF changes), further suggesting that this baseline value was erroneous. Because of the loss of data for two subjects, CBF data was analyzed separately $(n=6)$ from data for memory, sedation, plasma catecholamine, and plasma alprazolam level $(n=8)$.

\section{RESULTS}

As expected, plasma alprazolam levels rose significantly on both days following injection (Fig. 3), indicated by a significant time-interval effect $(F=12.5, p<0.0001$, Day $1 ; F=112.1, p<0.00001$, Day 8$)$. Peak levels oc. curred at the fifteen-minute time interval, and declined only slightly ( $10 \%$ to $30 \%$ ) over the next hour. Levels were higher at all times on Day 8 , and there was no significant interaction between time and day $(F=0.93$, $p=n s)$, indicating the absence of tolerance to the acute effect of plasma level itself.

Self-rated sedation also significantly increased over baseline (predrug) values on both days following al. 


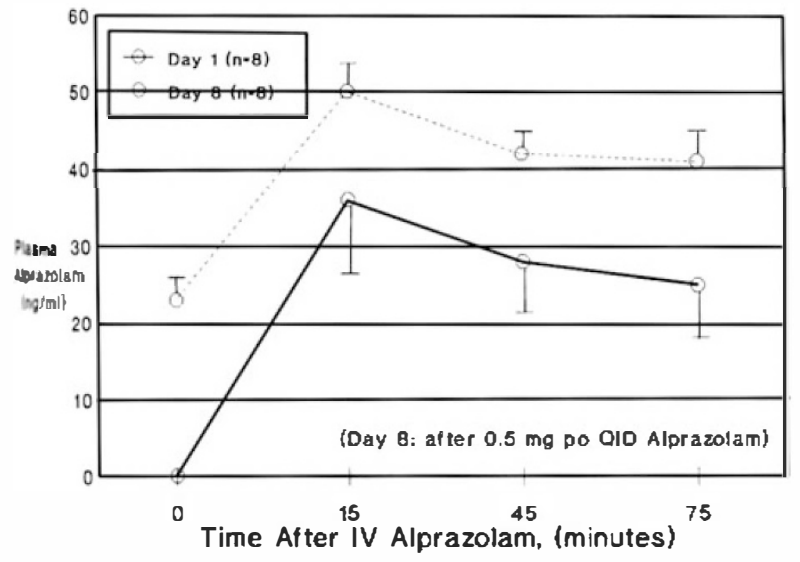

Fqure 3. Plasma alprazolam levels rise on both test days n a similar manner following IV injection of $0.014 \mathrm{mg} / \mathrm{kg}$ alprazolam $(F=36.6, p<0.0001)$. Levels are uniformly higher on Day 8 due to higher baseline steady-state alprazolam levds following $0.5 \mathrm{mg}$ po QID alprazolam treatment for 1 week.

prazolam $(F=14.5, p<0.00001$, Day $1 ; F=7.8, p<$ 0.001 , Day 8 ), without indications of tolerance (time interval $x$ day interaction: $F=0.53, p=n s$ ). Plasma norepinephrine showed such variability that there was no iqnificant effect of time interval on either day $(F=0.53$, $p=n s$, Day $1 ; F=0.47, p=$ ns, Day 8) and no indicaion of tolerance (time interval $\times$ day interaction: $F=$ $1.01, p=n s$ ), although levels decreased from baseline on Day 1 and slightly increased on Day 8.

Tolerance was observed, in contrast, for plasma epinephrine (Fig. 4) and for memory (Fig. 5). Plasma epinephrine significantly decreased from baseline (predrug) values on Day 1 (time-interval effect: $F=$ 13.24, $p<0.0001$ ), but not on Day 8 (time-interval effect: $\mathrm{F}=0.10, p=\mathrm{ns})$. Tolerance was suggested by a signifcant time-interval $\times$ day-interaction effect $(F=$ 7.7, $p$ <.001). Baseline values for plasma epinephrine were not significantly lower on Day $8(F=2.98, p=$ n). Memory performance (Fig. 5) significantly dereased from baseline (predrug) performance values on Day 1(time-interval effect: $F=10.0, p<0.001$ ), but not on Day 8 (time-interval effect: $F=1.5, p=n s$ ). Tolernce was suggested by a significant time-interval $\times$ dayinteraction effect $(F=3.5, p<0.05)$. Baseline values for nemory performance were highly similar on both days ( $\mathrm{F}=0.07, p=\mathrm{ns}$ ).

Table 1 displays the raw baseline and $\mathrm{CBF}$ values in each neuroanatomic region for both study days. A single $C B F$ value representing the largest decrease is employed because all three CBF values following alprazolam administration were similar to one another incomparison to the much higher baseline CBF value.

Table 2 displays $p$-values for time-interval effects (i.e., effects of acute alprazolam administration) on CBF in each neuroanatomic region (one-way repeated-

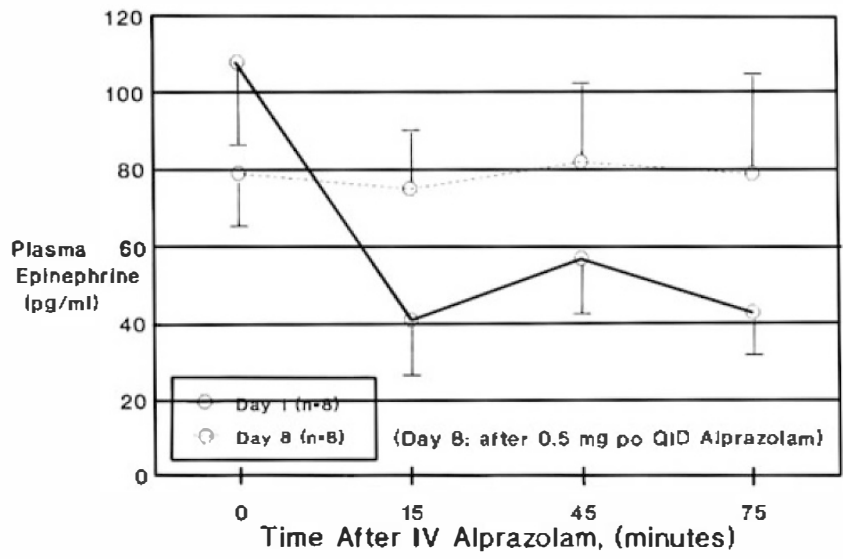

Figure 4. Alprazolam given acutely lowers plasma epinephrine on Day $1(F=13.24, p<0.0001)$. After 1 week of oral alprazolam treatment, plasma epinephrine is nonsignificantly lower at baseline and does not change with readministration of IV alprazolam on Day 8 ( $F=0.10, p=n s)$.

measures ANOVA: Day 1 and Day 8), for interaction effects between time interval and day (two-way ANOVA) (indicating differential acute alprazolam effects before and after chronic alprazolam treatment), and for differences in baseline CBF between Day 1 and Day 8 (ANOVA). On Day 1, absolute CBF values significantly decreased in all areas on slice 1 , in all areas but striatum on slice 2 , and only in the occipital lobes $(p<0.10)$ on slice 3. Decreases for whole-brain CBF ranging from $7 \%$ to $40 \%$ were significant on slices 1 and 2 (Figs. 6 and 7), and close to significant for slice 3 ( $p=$ 0.12 ). On Day 8, CBF values were not significantly decreased by the acute intravenous dose of alprazolam at the $p$ less than 0.05 level in any area and at the pless than . 10 level in only three areas. This lack of effect may

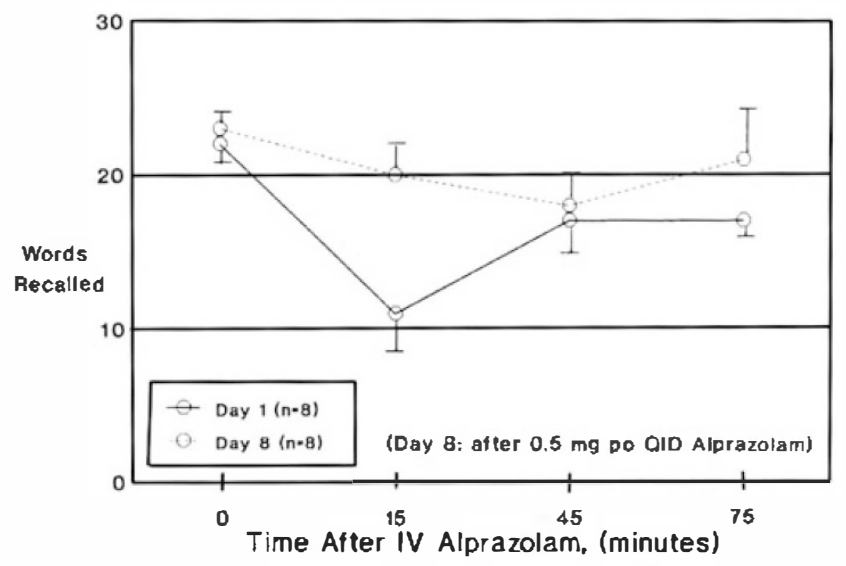

Figure 5. Alprazolam given acutely impairs memory on Day $1(F=10.0, p<0.001)$. After 1 week of oral alprazolam treatment, memory performance is similar at baseline and does not change significantly with readministration of IV alprazolam on Day $8(F=1.5, p=n s)$. 
Table 1. Regional $\mathrm{CBF}^{a}$ Effects of IV Alprazolam Before (Day 1) and After (Day 8) Chronic Alprazolam Treatment

\begin{tabular}{|c|c|c|c|c|}
\hline Tissue & $\begin{array}{c}\text { Predrug } \\
\text { Day } 1 \\
(\bar{X} \pm \text { SD) }\end{array}$ & $\begin{array}{c}\text { Postdrug } \\
\text { (Lowest } \\
\text { Value) } \\
\text { Day } 1 \\
(\bar{X} \pm \text { SD) }\end{array}$ & $\begin{array}{c}\text { Predrug } \\
\text { Day } 8 \\
(\bar{X} \pm \text { SD) }\end{array}$ & $\begin{array}{c}\text { Postdrug } \\
\text { (Lowest } \\
\text { Value) } \\
\text { Day } 8 \\
(\bar{X} \pm \text { SD) } \\
\end{array}$ \\
\hline \multicolumn{5}{|l|}{ SLICE 1} \\
\hline Cerebellum & $.63 \pm .30$ & $.37 \pm .07$ & $.53 \pm .28$ & $.44 \pm .21$ \\
\hline Left frontal & $.49 \pm .21$ & $.34 \pm .08$ & $.40 \pm .14$ & $.35 \pm .13$ \\
\hline Left occipital & $.50 \pm .17$ & $.35 \pm .05$ & $.45 \pm .12$ & $.39 \pm .10$ \\
\hline Left temporal & $.51 \pm .24$ & $.32 \pm .06$ & $.39 \pm .13$ & $.34 \pm .12$ \\
\hline Midbrain & $.52 \pm .25$ & $.34 \pm .07$ & $.42 \pm .19$ & $.36 \pm .18$ \\
\hline Right frontal & $.52 \pm .24$ & $.34 \pm .09$ & $.43 \pm .16$ & $.37 \pm .15$ \\
\hline Right occipital & $.50 \pm .20$ & $.35 \pm .04$ & $.42 \pm .09$ & $.39 \pm .09$ \\
\hline Right temporal & $.47 \pm .18$ & $.32 \pm .06$ & $.39 \pm .15$ & $.33 \pm .12$ \\
\hline Whole brain & $.49 \pm .21$ & $.33 \pm .06$ & $.41 \pm .15$ & $.35 \pm .14$ \\
\hline \multicolumn{5}{|l|}{ SLICE 2} \\
\hline Left frontal & $.38 \pm .13$ & $.28 \pm .04$ & $.31 \pm .10$ & $.29 \pm .08$ \\
\hline Left hipp/cing & $.44 \pm .14$ & $.31 \pm .06$ & $.35 \pm .10$ & $.33 \pm .09$ \\
\hline Left insula & $.47 \pm .12$ & $.34 \pm .07$ & $.38 \pm .08$ & $.35 \pm .10$ \\
\hline Left occipital & $.37 \pm .10$ & $.28 \pm .05$ & $.31 \pm .09$ & $.28 \pm .06$ \\
\hline Left parietal & $.45 \pm .22$ & $.29 \pm .06$ & $.36 \pm .17$ & $.31 \pm .11$ \\
\hline Left striatum & $.36 \pm .07$ & $.31 \pm .08$ & $.32 \pm .09$ & $.28 \pm 0.8$ \\
\hline Left thalamus & $.37 \pm .09$ & $.28 \pm .05$ & $.30 \pm .06$ & $.30 \pm .07$ \\
\hline Right frontal & $.38 \pm .13$ & $.28 \pm .04$ & $.32 \pm .11$ & $.29 \pm .10$ \\
\hline Right hipp/cing & $.42 \pm .15$ & $.31 \pm .06$ & $.35 \pm .11$ & $.34 \pm .08$ \\
\hline Right insula & $.46 \pm .12$ & $.35 \pm .07$ & $.38 \pm .12$ & $.34 \pm .12$ \\
\hline Right occipital & $.38 \pm .12$ & $.28 \pm .06$ & $.31 \pm .09$ & $.29 \pm .05$ \\
\hline Right parietal & $.43 \pm .21$ & $.27 \pm .05$ & $.34 \pm .16$ & $.30 \pm .12$ \\
\hline Right striatum & $.36 \pm .06$ & $.31 \pm .06$ & $.33 \pm .07$ & $.30 \pm .06$ \\
\hline Right thalamus & $.38 \pm .09$ & $.30 \pm .05$ & $.33 \pm .08$ & $.30 \pm .07$ \\
\hline Whole brain & $.40 \pm .12$ & $.30 \pm .04$ & $.32 \pm .07$ & $.32 \pm .07$ \\
\hline \multicolumn{5}{|l|}{ SLICE 3} \\
\hline Left frontal & $.39 \pm .13$ & $.28 \pm .02$ & $.38 \pm .16$ & $.31 \pm .08$ \\
\hline Left occipital & $.42 \pm .19$ & $.26 \pm .04$ & $.41 \pm .23$ & $.32 \pm .11$ \\
\hline Left parietal & $.33 \pm .09$ & $.26 \pm .04$ & $.36 \pm .15$ & $.30 \pm .11$ \\
\hline Right frontal & $.38 \pm .13$ & $.29 \pm .02$ & $.38 \pm .19$ & $.30 \pm .07$ \\
\hline Right occipital & $.39 \pm .17$ & $.26 \pm .04$ & $.38 \pm .21$ & $.31 \pm .10$ \\
\hline Right parietal & $.33 \pm .10$ & $.24 \pm .04$ & $.37 \pm .16$ & $.31 \pm .10$ \\
\hline Whole brain & $.38 \pm .13$ & $.27 \pm .02$ & $.37 \pm .15$ & $.32 \pm .08$ \\
\hline
\end{tabular}

" $\mathrm{CBF}$ is given in milligrams per gram per minute.

be partially the result of significantly lower baseline CBF values on Day 8 that were significantly lower in all areas of slice 2 and nonsignificantly lower in all areas of slice 1 (i.e., a "floor" effect). There was, as would be expected from the univariate ANOVA's on Days 1 and 8, a suggestion of tolerance to benzodiazepine effects on CBF, with a trend for time interval $x$ day interaction for whole brain on slices 1 and 2 (Figs. 6 and 7). Although a few individual neuroanatomic regions showed evidence $(p<0.10)$ of interaction, the majority of areas on both slices 1 and 2 had low but nonsignificant interaction $p$-values ( 0.10 to 0.20$)$, and all areas on slice 3 had high $p$-values ( 0.36 to 0.67$)$. This suggested that tolerance to the benzodiazepine effect on CBF is a general phenomenon affecting each slice, without a specific focus in any particular region. Therefore, additional
ANOVAs were performed on regional CBF values normalized for the respective whole-slice CBF (region whole-slice). This analysis gave no evidence of any significant regional tolerance; moreover, it eliminated all previously significant acute effects as well $(p>0.40 \mathrm{for}$ all analyses), suggesting that acute regional effects were all components of a more global effect on brain CBF.

There were no correlations between alprazolaminduced changes in any of the variables on Day 1, Day 8 , or the Day 8 minus Day 1 difference.

\section{DISCUSSION}

In this study, intravenous administration of a fairly large, sedating dose of alprazolam resulted in acute 
Table 2. Alprazolam Effects on CBF: $p$-Values for ANOVAs

\begin{tabular}{|c|c|c|c|c|}
\hline Tissue & $\begin{array}{l}\text { Within } \\
\text { Session } \\
\text { (Day 1) }\end{array}$ & $\begin{array}{l}\text { Within } \\
\text { Session } \\
\text { (Day 8) }\end{array}$ & $\begin{array}{c}\text { Within } \times \\
\text { Between } \\
\text { Session } \\
\text { Interaction }\end{array}$ & $\begin{array}{c}\text { Baseline } \\
\text { (Day } 1 \text { vs. } \\
\text { Day 8) }\end{array}$ \\
\hline \multicolumn{5}{|l|}{ SLICE 1} \\
\hline Cerebellum & .02 & .20 & .18 & .33 \\
\hline Left frontal & .01 & .09 & .14 & .21 \\
\hline Left occipital & .02 & .34 & .11 & .40 \\
\hline Left temporal & .01 & .21 & .06 & .17 \\
\hline Midbrain & .02 & .15 & .07 & .11 \\
\hline Right frontal & .02 & .08 & .17 & .27 \\
\hline Right occipital & .04 & .85 & .15 & .28 \\
\hline Right temporal & .01 & .14 & .07 & .13 \\
\hline Whole brain & .01 & .10 & .09 & .19 \\
\hline \multicolumn{5}{|l|}{ SLICE 2} \\
\hline Left frontal & .06 & .69 & .23 & .01 \\
\hline Left hipp/cing & .01 & .74 & .03 & .06 \\
\hline Left insula & .02 & .70 & .17 & .02 \\
\hline Left occipital & .02 & .53 & .09 & .03 \\
\hline Left parietal & .05 & .53 & .14 & .01 \\
\hline Left striatum & .16 & .32 & .34 & .08 \\
\hline Left thalamus & .03 & .95 & .15 & .01 \\
\hline Right frontal & .04 & .59 & .16 & .003 \\
\hline Right hipp/cing & .07 & .89 & .14 & .12 \\
\hline Right insula & .05 & .49 & .34 & .05 \\
\hline Right occipital & .05 & .60 & .16 & .05 \\
\hline Right parietal & .03 & .65 & .08 & .01 \\
\hline Right striatum & .31 & .54 & .60 & .27 \\
\hline Right thalamus & .03 & .85 & .22 & .03 \\
\hline Whole brain & .03 & .94 & .08 & .04 \\
\hline \multicolumn{5}{|l|}{ SLICE 3} \\
\hline Left frontal & .13 & .11 & .56 & .94 \\
\hline Left occipital & .06 & .16 & .37 & .85 \\
\hline Left parietal & .24 & .07 & .67 & .69 \\
\hline Right frontal & .16 & .18 & .58 & .93 \\
\hline Right occipital & .10 & .19 & .36 & .87 \\
\hline Right parietal & .14 & .11 & .60 & .47 \\
\hline Whole brain & .12 & .19 & .66 & .100 \\
\hline
\end{tabular}

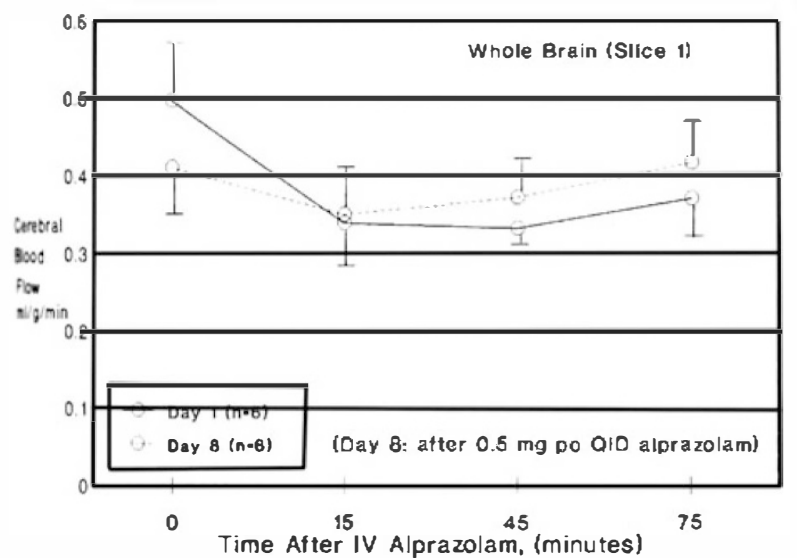

Fare 6. Alprazolam given acutely lowers whole-brain $\mathrm{CBF}$ in cortical areas on Day $1(F=4.54, p<0.01)$. After 1 week doral al prazolam treatment, blood flow is nonsignificantly bwer at baseline and does not change significantly with readinistration of IV alprazolam on Day $8(F=2.41, p=0.11)$.

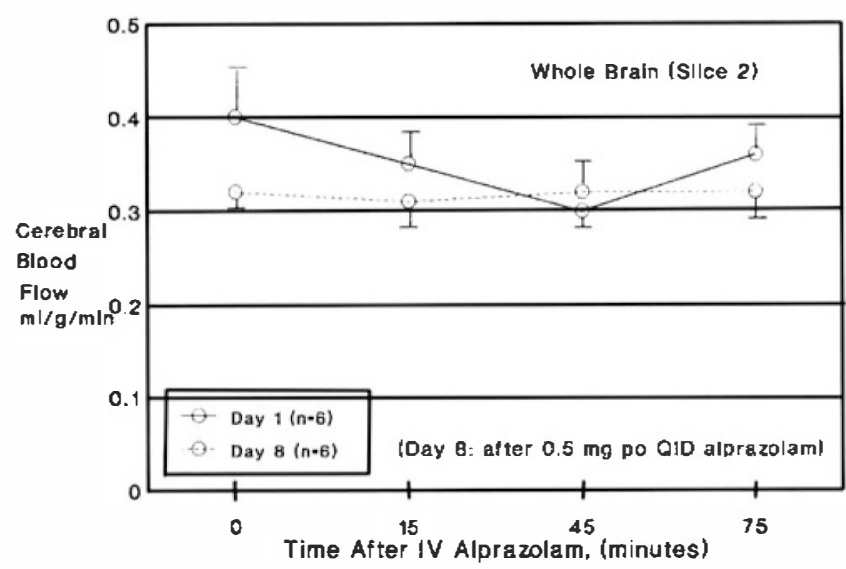

Figure 7. Alprazolam given acutely lowers whole-brain $\mathrm{CBF}$ in subcortical areas on Day $1(F=3.89, p=0.03)$. After 1 week of oral alprazolam treatment, blood flow is significantly lower at baseline (time 0$)(F=7.01, p=0.04)$ and does not change significantly with readministration of IV alprazolam on Day $8(F=0.13, p=0.94)$. 
decreases in memory performance, plasma epinephrine, whole-brain $C B F$, and self-rated alertness. After 1 week of oral alprazolam treatment at standard anxiolytic doses ( $2 \mathrm{mg} /$ day), tolerance developed to the acute alprazolam decreases on the first three measures, but not to the sedative effects, using a crude self-report measure. However, all subjects definitely appeared less sedated behaviorally during the second acute study. Furthermore, tolerance to the sedative effects of alprazolam has been previously observed following 3 days of administration of $3 \mathrm{mg}$ and $6 \mathrm{mg}$ daily doses in healthy volunteers (Fleishaker et al. 1989).

Changes in CBF following acute alprazolam treatment appeared fairly uniform throughout the brain regions sampled, and tolerance on acute rechallenge after a 1-week treatment was suggested by data from slices 1 and 2. No specific regional focus of the CBF effect or tolerance to it was indicated after normalizing CBF values to whole-brain (slice) $\mathrm{CBF}$. All regional effects evident when the regions were analyzed separately disappeared after the values were divided by the $C B F$ value of the corresponding slice, confirming that such effects were entirely attributable to changes in global CBF. We did not observe the consistent difference between cortical and subcortical benzodiazepine effects observed by de Witt et al. (1991), although the only region in slice 2 not showing acute effects was the striatum. Regional differences following acute diazepam noted by one group (Mathew et al. 1986) were not replicated by this same group (Mathew et al. 1991) when a $20 \%$ higher dose of diazepam was injected rapidly (over 1 minute as in our study) instead of more slowly (over 5 minutes). It is possible that regional differences in this study might have been more apparent with a lower dose of alprazolam.

The decrease in whole-brain CBF seen with acutely administered alprazolam is interestingly similar to the decrease in whole-brain glucose metabolism seen following cocaine administration (London et al. 1990). Since glucose metabolism is usually, although not always, tightly coupled to blood flow, and since alprazolam is also a euphoriant with addictive potential in vulnerable individuals (Ciraulo et al. 1988), this is consistent with the suggestion by London et al. that euphoriant and/or addictive drugs may lower wholebrain metabolism.

Following 1 week of alprazolam treatment, decreases in baseline CBF were present throughout slice 2 , suggesting that tolerance may have been in part a "floor" effect. Although this might be due to oral alprazolam treatment already producing near-maximum CBF decreases in some patients, it is also possible that lower CBF during the second scan was due to lower patient stress, greater familiarity with the procedure, or the antianxiety effects of alprazolam. A placebo group or cross-over design would be required to attribute this definitively to maximal alprazolam effects. The reason for failure to find differences in baseline CBF values on slices 1 and 3 is unclear but it might be related either to true neuroanatomic differences, or to technical variation in data acquisition/reconstruction with different scanner slices (slice 2 was the cross-plane slice).

Our absolute $\mathrm{CBF}$ values were somewhat lower than those usually reported in the literature. This may be due to our relatively crude method of scatter correction. Nonetheless, all values were similarly determined and were quite consistent for individual subjects across regions and slices. Failure to find correlations between different benzodiazepine effects across individuals is not unusual, even in samples of far greater size than ours. Although a within-subjects analysis can demonstrate a statistically significant correlation (Bond and Lader 1983), individual variability of baseline levels and a nonlinear response due to "floor" and "ceiling" effects often prevents between-subjects correlations from reaching significance.

In conclusion, this study has demonstrated that tolerance to the effects of acutely administered alprazolam develops in normal subjects after 1 week of continuous treatment with anxiolytic doses of oral alprazo. lam. Tolerance appeared to be nonspecifically present across multiple pharmacodynamic measures and was evident even without controlling for the higher alprazo. lam blood levels that subjects had on the second test day. No evidence of regional neuroanatomic differences in either acute alprazolam effects on CBF or regional differences in tolerance to these acute effects as has been seen in preclinical studies (Laurie and Pratt 1989) was evident, perhaps due to the higher acute doses em. ployed.

\section{ACKNOWLEDGMENT}

This study was approved by the University of Washington Human Subjects and the Radiation Safety Committees and supported by a grant from the UpJohn Company.

\section{REFERENCES}

Ableitner A, Wüster M, Herz A (1985): Specifıc changes in local cerebral glucose utilization in the rat brain induced by acute and chronic diazepam. Brain Res 359-356

Bond A, Lader M (1983): Correlations among measures of re. sponse to benzodiazepines in man. Pharmacol Biochem Behav 18:295-298

Bond AJ, Lader MH (1974): The use of analogue scales in rat. ing subjective feelings. Br J Med Psychol 47:211-218

Brahams D (1990): Benzodiazepine over-prescribing: successful initiative in New York State. Lancet 336:1372-1373

Buchsbaum MS, Wu J, Haier R, Hazlett E, Ball R, Katz M. Sokolski K, Lagunas-Solar M, Langer D (1987): Positron 
emission tomography assessment of effects of benzodiazepines on regional glucose metabolic rate in patients with anxiety disorder. Life Sci 40:2393-2400

Craulo DA, Bainhill JG, Greenblatt DJ, Shader RI, Ciraulo AM, Tarmey MF, Molloy MA, Foti ME (1988): Abuse liability and clinical pharmacokinetics of alprazolam in alcoholic men. J Clin Psychiatry 49:333-337

deWitt H, Metz J, Wagner N, Cooper M (1991): Effects of diazepam on cerebral metabolism and mood in normal volunteers. Neuropsychopharmacology 5:33-34

Dura R, Gross-Glenn K, Barker WW, Chang JY, Apicella A, Loewenstein D, Boothe T (1987): Behavioral activation and the variability of cerebral glucose metabolic measurements. J Cereb Blood Flow Metab 7:266-271

Evans Ml, Holter JB, Porte D (1987): Comparison of doubleand single-blind isotope derivative methods for measuring catecholamines in human plasma. Clin Chem 24:567-570

Reishaker JC, Phillips JP, Eller MG, Smith RB (1989): Pharmacokinetics and pharmacodynamics of alprazolam following single and multiple oral doses of a sustained release formulation. J Clin Pharmacol 29:543-549

Gaham MM, Lewellen BL (1988): High speed automated discrete blood sampling for PET. J Nucl Med 29:879-882

Hesscovitch P, Markham J, Raichle ME (1984): Brain blood flow measured with intravenous $\mathrm{H}_{2}{ }^{15} \mathrm{O}$ I. Theory and error analysis. J Nucl Med 24:782-789

Lurie DJ, Pratt JA (1989): Local cerebral glucose utilization following subacute and chronic diazepam treatment: Differential tolerance. Brain Res 504:101-111

London ED, Cascella NG, Wong DF, Phillips RL, Dannals
RF, Links JM, Herning R, Grayson R, Jaffe JH, Wagner HN (1990): Cocaine-induced reduction of glucose utilization in human brain: a study using positron emission tomography and (fluorine 18)-fluorodeoxyglucose. Arch Gen Psychiatry 47:567-576

Mathew RJ, Wilson WH (1991): Evaluation of the effects of diazepam and an experimental anti-anxiety drug on regional cerebral blood flow. Psychiatry Res: Neuroimaging 40:125-134

Mathew RJ, Wilson WH, Daniel DG (1985): The effect of nonsedating doses of diazepam on regional cerebral blood flow. Biol Psychiatry 20:1109-1116

McLeod DR, Hoehn-Saric R, Labib AS, Greenblatt DJ (1988): Six weeks of diazepam treatment in normal women: Effects on psychomotor performance and psychophysiology. J Clin Psychopharmacol 8:83-99

Nehlig A, Daval J-L, deVasconcelos AP, Boyet S (1987): Caffeine-diazepam interaction and local cerebral glucose utilization in the conscious rat. Brain Res 419:272-278

Roy-Byrne P, Nutt DJ (1991): Benzodiazepines: biologic mechanisms. In Roy-Byrne PP, Cowley DS (eds), Benzodiazepines in Clinical Practice: Risks and Benefits. Washington, DC, APA Press, pp 5-18

Roy-Byrne PP, Uhde TW, Holcomb HH, King AK, Thompson K, Weingartner $\mathrm{H}$ (1987): Effects of diazepam on cognitive processes in normal subjects. Psychopharmacology 91:30-33

Smith RB, Kroboth PD (1987): Influence of dosing regimen on alprazolam and metabolite serum concentrations and tolerance to sedative and psychomotor effects. Psychopharmacology 93:105-112 